\title{
Functional Outcomes After Critical Pathway for Inpatient Rehabilitation of Total Knee Arthroplasty
}

\author{
Jong Hyun Kim, MD¹, Bo Ryun Kim, MD, PhD ${ }^{1}$, Sang Rim Kim, MD, PhD ${ }^{2}$, Eun Young Han, MD, PhD ${ }^{1}$, \\ Kwang Woo Nam, MD, $\mathrm{PhD}^{2}$, So Young Lee, $\mathrm{MD}^{1}$, Won Bin Kim, $\mathrm{MD}^{1}$
}

\begin{abstract}
${ }^{1}$ Department of Rehabilitation Medicine, Jeju National University College of Medicine-Regional Rheumatoid and Degenerative Arthritis Center, Jeju National University Hospital, Jeju; ${ }^{2}$ Department of Orthopedic Surgery, Jeju National University College of Medicine-Regional Rheumatoid and Degenerative Arthritis Center, Jeju National University Hospital, Jeju, Korea
\end{abstract}

Objective To investigate functional outcomes after the application of a critical pathway for inpatient rehabilitation of total knee arthroplasty (TKA).

Methods A total of 184 patients (57 males and 127 females; average age, $71.5 \pm 5.9$ years) who underwent unilateral or bilateral TKA were included. The critical pathway included early, intensive individualized rehabilitation exercises. Patients completed the following performance-based physical function tests: the stair climbing test (SCT), 6-minute walk test (6MWT), and Timed Up and Go test (TUG) as well as measurement of isometric knee flexor and extensor strength of the operated knee, gait speed, and range of knee flexion and extension. Self-reported physical function and pain were measured using the Western Ontario McMaster Universities Osteoarthritis Index (WOMAC) and visual analog scale (VAS), respectively, and self-reported quality of life was measured using the EuroQoL 5 dimension (EQ-5D) questionnaire. These evaluations were performed preoperatively and at 1 month and 3 months postoperatively.

Results Performance-based and self-reported physical function and quality of life measures improved nonlinearly over time. Specifically, the 6WMT, TUG, gait speed, WOMAC-pain, WOMAC-function, VAS, and EQ-5D scores showed a significant improvement at 1-month post-TKA, whereas SCT, peak torque of the knee extensors and flexors, and WOMAC-stiffness scores showed gradual, but substantial, improvements over 3 months. There were between-group differences (unilateral and bilateral TKA groups) in the time course of the SCT, 6MWT, TUG, VAS, WOAMC-stiffness, and WOMAC-function results.

Conclusion Patients who underwent critical pathway rehabilitation after TKA showed significant improvements in functional measurements during the first 3 months post-surgery.

Keywords Arthroplasty, Knee, Osteoarthritis, Rehabilitation, Critical pathways

Received March 28, 2019; Accepted July 2, 2019

Corresponding author: Bo Ryun Kim

Department of Rehabilitation Medicine, Jeju National University Hospital, 15 Aran 13-gil, Jeju 63241, Korea. Tel: +82-64-717-2711, Fax: +82-64-7171131, E-mail: brkim08@jejunu.ac.kr

ORCID: Jong Hyun Kim (http://orcid.org/0000-0001-7314-6500); Bo Ryun Kim (http://orcid.org/0000-0001-7788-7904); Sang Rim Kim (http://orcid. org/0000-0001-5814-1031); Eun Young Han (http://orcid.org/0000-0002-4780-8862); Kwang Woo Nam (http://orcid.org/0000-0003-1096-149X); So Young Lee (http://orcid.org/0000-0002-0433-4548); Won Bin Kim (http://orcid.org/0000-0003-3868-5247).

(c) This is an open-access article distributed under the terms of the Creative Commons Attribution Non-Commercial License (http://creativecommons.org/ licenses/by-nc/4.0) which permits unrestricted noncommercial use, distribution, and reproduction in any medium, provided the original work is properly cited. Copyright ( 2019 by Korean Academy of Rehabilitation Medicine 


\section{INTRODUCTION}

Knee osteoarthritis (OA) is one of the most common joint disorders and a leading cause of joint pain, swelling, functional limitation, and poor quality of life in the elderly [1]. Total knee arthroplasty (TKA) is the standard treatment for severe knee osteoarthritis, with demonstrable benefits in terms of improving pain, activity, and quality of life [2-4]. An increasing number of patients undergo TKA each year, therefore, it is important to identify appropriate rehabilitation programs after surgery; indeed, physical rehabilitation programs are widely advocated and are provided routinely.

TKA reduces pain and improves quality of life; studies have shown that fast-track surgery and an enhanced postoperative recovery program improve the initial postoperative physical performance, reduce the length of hospital stay from 4 to 2 days after surgery, and make readmission less likely [5-7]. However, quadriceps muscle strength is $41 \%$ weaker, walking distance is $28 \%$ lower, stair-climbing speed is $105 \%$ slower, and active knee flexion and extension are reduced after TKA, despite patients undergoing standard rehabilitation protocols $[8,9]$. Moreover, a randomized controlled trial of 165 TKA patients showed no difference in terms of mobility between a home-based only group and a 10-day admission rehabilitation service group at the 26th week assessment [10]. In addition, there was a critical pathway for rehabilitation after hip fracture surgery in South Korea [11] but lack of that for postTKA rehabilitation. Most studies included outpatients or inpatients for a short-term period (3-4 days) after TKA, had inadequate accurate and detailed rehabilitation protocols, and did not report varied evaluation outcomes [12-16]. Therefore, more effective post-TKA rehabilitation protocols are needed. At present, there is no critical pathway for intensive rehabilitation during the early post-TKA period in South Korea. Therefore, the aim of this study was to evaluate short-term functional outcomes after intensive critical pathway rehabilitation of inpatients after TKA.

\section{MATERIALS AND METHODS}

\section{Participants}

This retrospective cohort study included 184 patients (158 females and 26 males; average age, 71.5 \pm 5.9 years) who were diagnosed with end-stage primary knee OA. A total of 1,203 patients diagnosed with end-stage primary knee OA were recruited. We excluded 584 patients who were not evaluated preoperatively. Further inclusion criteria were as follows: (1) be scheduled for a first TKA, (2) hospitalized between October 2013 and April 2018, and (3) could walk independently with or without an ambulatory aid. A total of 227 patients were excluded as they underwent TKA surgery at another hospital, as were 208 patients who did not complete the 3-month follow-up. Finally, 184 patients were enrolled in the study (Fig. 1). The study protocol was approved by the local ethics committee of Jeju National University Hospital (No. 2019-01015). Written informed consents were obtained.

\section{The critical pathway}

When the need for an operation was confirmed, the orthopedic surgery department referred the patient to the rehabilitation department for preoperative consultation and evaluation, which included performancebased physical function tests and self-reported physical function questionnaires. If vital signs were stable after TKA, the patient was started on an early rehabilitation protocol, which comprised range of motion (ROM) exercises using a continuous passive motion device and progressive resistance exercises (incline leg press, leg press, leg extension, leg curl, hip abduction, and hip adduction) using air resistance machines (HUR Co., Kok-

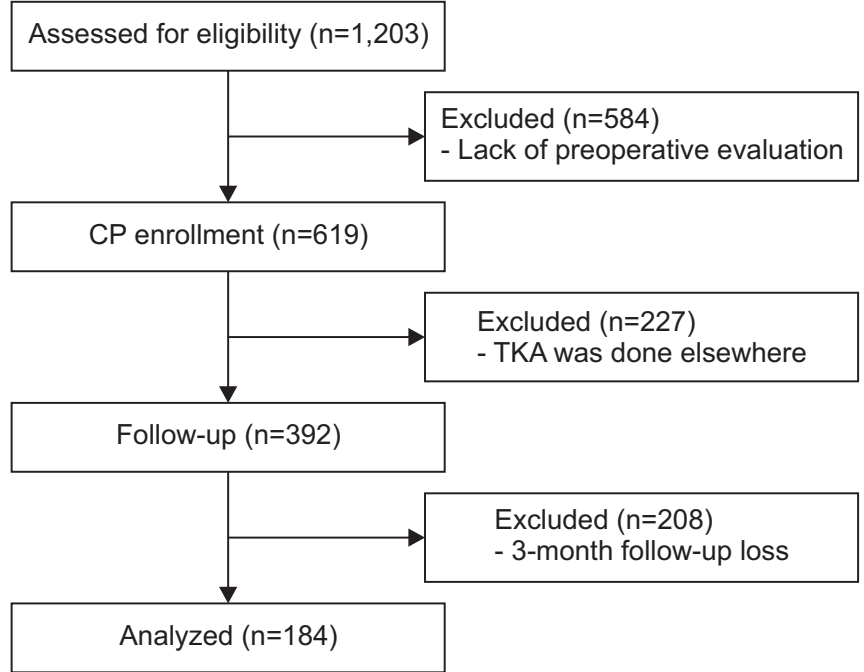

Fig. 1. Flow chart of patient inclusion with reason for exclusion and total study population. $\mathrm{CP}$, critical pathway; TKA, total knee arthroplasty. 

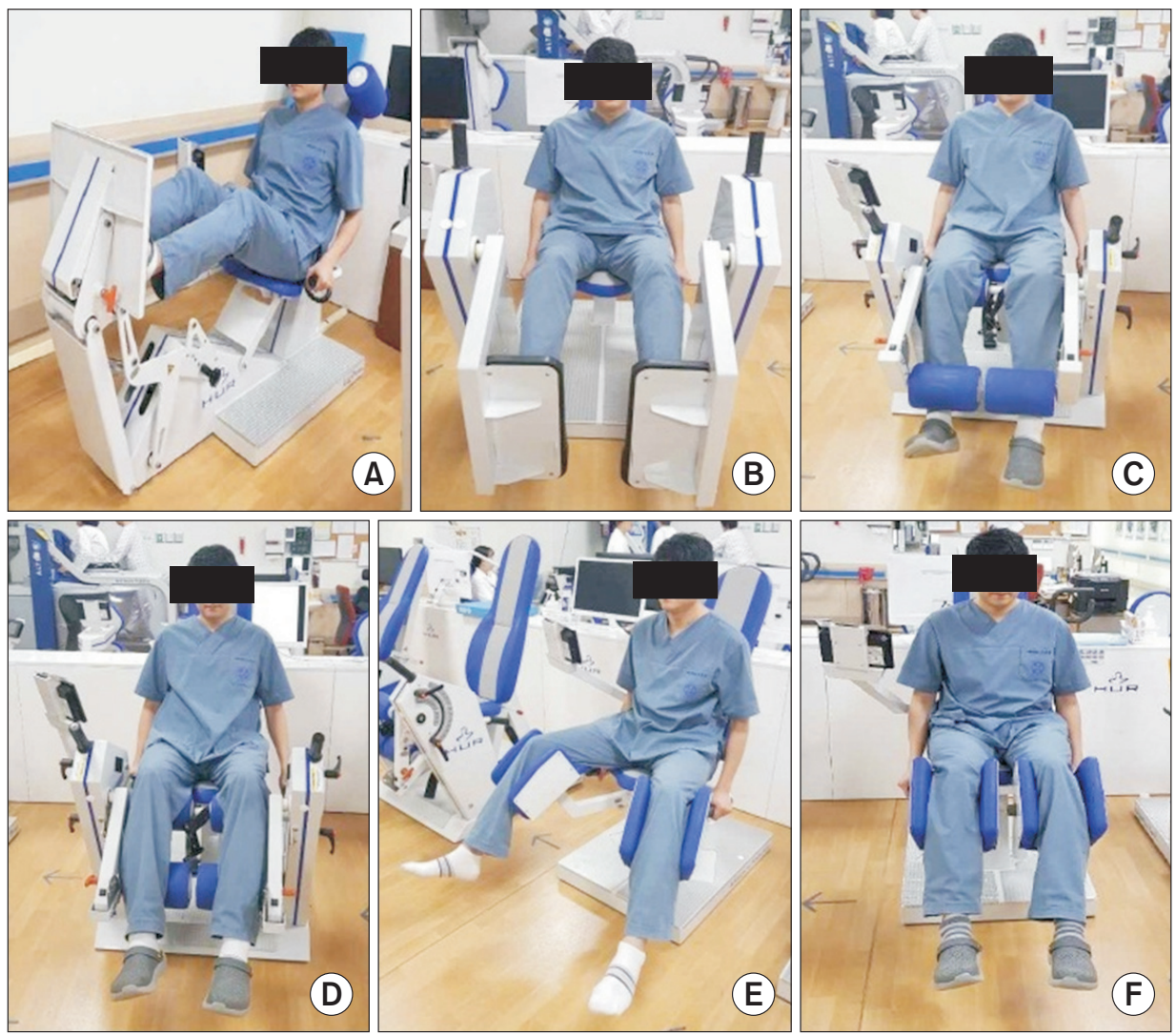

Fig. 2. The progressive resistance exercises consisted of (A) incline leg press, (B) leg press, (C) leg extension, (D) leg curl, (E) hip abduction, and (F) hip adduction using air-resistance machines (HUR Co., Kokkola, Finland). kola, Finland) (Fig. 2). The 1 repetition maximum (1RM) for each patient was measured. Resistance loads began at $10 \%-20 \%$ of the 1RM ( 3 sets of 15 repetitions) from the 4 th day post-surgery. Two weeks post-surgery, the patient was transferred to the rehabilitation department.

The intensive rehabilitation program included progressive gait training using a lower-body positive pressure treadmill (Alter-G Inc., Fremont, CA, USA) (Fig. 3), aerobic exercise using a stationary ergometer, and progressive resistance exercises. Progressive resistance exercises using the HUR increased gradually from $30 \%$ (3 sets of 15 repetitions) to $60 \%$ of $1 \mathrm{RM}$; the number of repetitions decreased as the load increased.

For progressive gait training, patients began with $50 \%$ bodyweight support (BWS) and walked at a speed of 2.0 $\mathrm{km} / \mathrm{hr}$; BWS was reduced gradually. When BWS was no longer needed, patients moved onto a land treadmill. They received stair training on the day of discharge. The G-trainer was used daily, whereas the ergometer and HUR machine were used on alternate days. The TKA rehabilitation programs were performed as follows: 30 minutes per session, 2 sessions per day, 5 days per week, for 2 weeks.

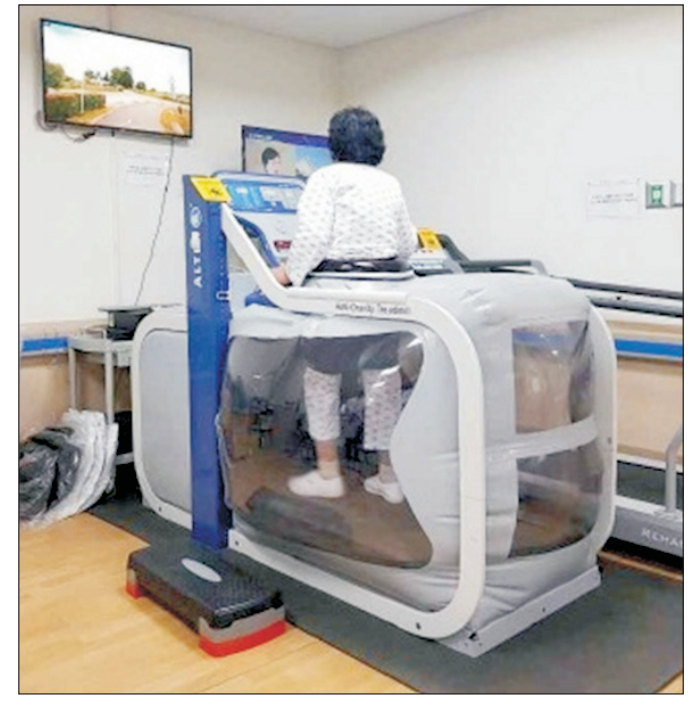

Fig. 3. The gait training exercises consisted of Alter G anti-gravity treadmill (Model M320; Alter G Inc., Fremont, CA, USA).

To enable self-exercises in the ward, the rehabilitation nurse instructed patients how to perform a range of joint motion and TheraBand exercises; patients were provided with an exercise brochure. Prior to discharge, patients 
were educated about self-exercise for home-based rehabilitation. Evaluations including performance-based physical function and self-reported physical function were performed 1 month and 3 months post-surgery (Fig. 4).

\section{Outcome measures}

Each examination was performed preoperatively, 1 month postoperatively, and 3 months postoperatively, giving sufficient rest time; all outcome measures were conducted on 1 day. In all patients, isometric strength of the knee extensors and flexors was measured by a physical therapist, and other tests, including the questionnaire, were performed by the education nurse at our arthritis center.

\section{Performance-based physical function tests}

\section{Stair climbing test}

The stair climbing test (SCT) was used to assess functional mobility and the ability of the lower extremity muscles to generate concentric and eccentric forces. For the test, the time taken to ascend and descend a flight of stairs (12 steps; each step was $17-\mathrm{cm}$ high and $25-\mathrm{cm}$ wide) was measured [17].

\section{Six-minute walk test}

The 6-minute walk test (6MWT), a measure of the distance $(\mathrm{m})$ that an individual walks in 6 minutes, was used to assess the overall locomotor ability and locomotor fatigue. Patients were instructed to walk as far as possible for 6 minutes along a 50-m hallway that was marked with lines [18].

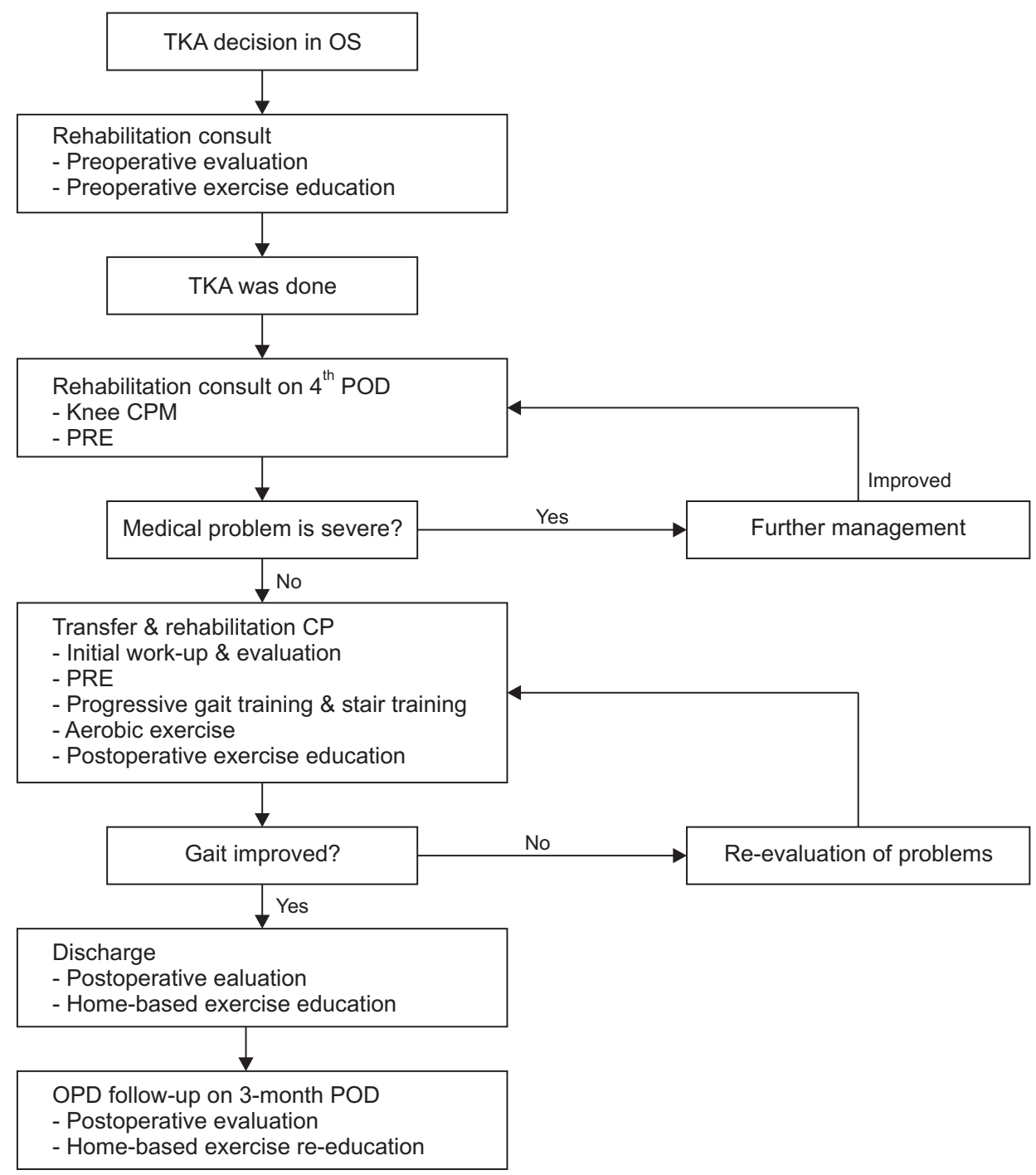

Fig. 4. Critical pathway after total knee arthroplasty. OS, orthopedic surgeon; TKA, total knee arthroplasty; POD, post-operation day; $\mathrm{CPM}$, continuous passive motion; $\mathrm{CP}$, critical pathway; PRE, progressive resistance exercise; OPD, outpatient department. 


\section{Timed Up and Go test}

For the Timed Up and Go (TUG) test, patients began in a seated position in an armchair (seat height, $44 \mathrm{~cm}$; depth, $45 \mathrm{~cm}$; width, $49 \mathrm{~cm}$; and armrest height, $64 \mathrm{~cm}$ ). They were then instructed to rise, walk forward for $3 \mathrm{~m}$, turn around, walk back to the chair, and sit back down without any physical assistance. The whole process was timed and evaluated the dynamic balance [19].

\section{Isometric strength of the knee flexors and extensors}

The maximal isometric strength of the bilateral knee extensors and flexors was measured using an isokinetic dynamometer (Computer Sports Medicine Inc., Stoughton, MA, USA). Before the test, all patients relaxed their muscles by performing light stretches [20]. Patients were instructed to grasp the sidebars of the apparatus during the test procedure. After a structured warm-up with the knee joint fixed at $60^{\circ}$ of flexion (to generate maximal isometric force) [21], patients were instructed to perform maximal voluntary contractions until the torque did not increase by $>5 \%$ on three successive attempts.

\section{Gait speed}

Spatiotemporal variables of gait were measured using a validated wireless inertial sensing device (BTS Bioengineering S.p.A., Milan, Italy). The first and last steps of the 8-m walk were removed from all calculations to eliminate the effects of abnormal patterns during the initiation of gait or psychological responses at the termination of gait [22].

\section{Measurement of knee ROM}

Available knee ROM was measured using a standard long arm goniometer. Knee flexion was measured as the value of maximal active bend at the knee while the patient was lying in a supine position. Knee extension was measured as the angle of maximal active straightening with the patient's heel placed on a $10-\mathrm{cm}$ wooden block [23].

Self-reported physical function, pain, and quality of life

The Western Ontario McMaster Universities Osteoarthritis Index

The multidimensional Western Ontario McMaster Universities Osteoarthritis Index (WOMAC) questionnaire includes questions on pain, stiffness, and physical func- tion. The questionnaire includes 5 pain, 2 stiffness, and 17 physical function variables. Each of these variables is scored on the Likert scale ( 0 , none; 1 , slight; 2 , moderate; 3 , very; and 4, extremely), which is used to measure outcomes in rheumatology clinical trials. Thus, the Likert scale determines the degree of pain, stiffness, and difficulty experienced when performing each of the 17 activities during the preceding 48 hours. Higher scores indicate greater levels of pain, stiffness, and difficulty [24].

\section{Visual analog scale}

Patients were instructed to evaluate their level of knee pain using visual analog scale (VAS), which was measured when the patient was resting. The scale consisted of a 10-cm long horizontal line that ranged from 'no pain' to 'worst possible pain'. The VAS system ranged from 0 (no pain) to 10 (worst possible pain) points [25].

\section{EuroQoL 5 dimension questionnaires}

The EuroQoL 5 dimension (EQ-5D) questionnaire is used to evaluate self-reported quality of life. The EQ-5D index is used widely to measure general health status. The instrument comprises questionnaires with the following 5 dimensions: mobility, self-care, usual activities, pain/discomfort, and anxiety/depression. Each dimension is represented by one question that has three severity levels, namely, no problems, some or moderate problems, and extreme problems [26].

\section{Statistical analysis}

All statistical analyses were performed using IBM Statistical Package for the Social Sciences software version 18.0 (IBM Co., Armonk, NY, USA). All variables were subjected to descriptive statistics. Repeated-measures ANOVA was performed to assess the effect of time on the postoperative outcomes of performance-based and selfreported physical function and on the quality of life. Posthoc analyses using Bonferroni test were performed to detect significant interactions between group and time. A p-value of $<0.05$ was considered significant.

\section{RESULTS}

Patient demographics and characteristics are presented in Table 1. There were 158 (85.9\%) females and 26 (14.1\%) males (average age, $71.5 \pm 5.9$ years). The average body 
Table 1. Demographic and disease-related characteristics of the subjects $(\mathrm{n}=184)$

\begin{tabular}{|lc|}
\hline \multicolumn{1}{|c}{ Characteristic } & Value \\
\hline Age $(\mathrm{yr})$ & $71.5 \pm 5.9$ \\
\hline Sex & $26(14.1)$ \\
\hline Male & $158(85.9)$ \\
\hline Female & $26.1 \pm 3.1$ \\
\hline BMI $\left(\mathrm{kg} / \mathrm{m}^{2}\right)$ & \\
\hline Type of surgery & $127(69.0)$ \\
\hline Unilateral & $57(31.0)$ \\
\hline Bilateral & \\
\hline K-L grades & $34(14.1)$ \\
\hline Grade 3 & $207(85.9)$ \\
\hline Grade 4 & \\
\hline Lesion side & $135(56.0)$ \\
\hline Right & $106(44.0)$ \\
\hline Left & \\
\hline Comorbidities & $125(67.9)$ \\
\hline Hypertension & $35(19.0)$ \\
\hline Diabetes mellitus & $24(13.0)$ \\
\hline Degenerative spine disease & $93(50.5)$ \\
\hline Osteoporosis & $10(5.8)$ \\
\hline Presarcopenia & \\
\hline
\end{tabular}

Values are presented as mean \pm standard deviation or number (\%).

BMI, body mass index; K\&L, Kellgren-Lawrence.

mass index was $26.1 \pm 3.1 \mathrm{~kg} / \mathrm{m}^{2}$. Of the 184 patients, 127 (69\%) underwent unilateral TKA and the remaining 57 (31\%) underwent bilateral TKA.

Postoperative outcomes with respect to objective performance-based physical function, self-reported physical function, pain, and quality of life

Postoperative outcomes with respect to performancebased physical function, self-reported physical function, pain, and quality of life are presented in Table 2.

The performance-based and self-reported physical function and quality of life measures improved nonlinearly over time. Specifically, the 6MWT (314.48 \pm 100.60 vs. $366.65 \pm 81.40$ vs. $444.04 \pm 95.36 \mathrm{~m} ; \mathrm{p}<0.001)$, TUG test $(12.00 \pm 3.45$ vs. $11.28 \pm 2.94$ vs. $9.31 \pm 1.75$ seconds; $\mathrm{p}<$ $0.001)$, gait speed $(0.89 \pm 0.17$ vs. $1.02 \pm 0.15$ vs. $1.22 \pm 0.76$ $\mathrm{m} / \mathrm{s} ; \mathrm{p}<0.001)$, WOMAC-pain $(9.58 \pm 3.16$ vs. $57 \pm 2.5$ vs. $4.7 \pm 2.15 ; \mathrm{p}<0.001)$, WOMAC-function $(29.58 \pm 8.83$ vs.
$26.29 \pm 8.54$ vs. $18.72 \pm 8.16 ; \mathrm{p}<0.001)$, VAS $(6.98 \pm 1.70$ vs. $3.67 \pm 1.21$ vs. $2.50 \pm 2.21 ; \mathrm{p}<0.001)$, and EQ-5D $(0.58 \pm 0.15$ vs. $0.73 \pm 0.06$ vs. $0.81 \pm 0.08 ; \mathrm{p}<0.001)$ scores improved significantly between the preoperative, 1 month postoperative, and 3 months postoperative measurements, respectively. The SCT ascent ( $14.14 \pm 5.71$ vs. $12.13 \pm 4.03$ seconds; $\mathrm{p}<0.001)$, SCT descent $(16.90 \pm 5.81$ vs. $12.13 \pm 4.03$ seconds; $\mathrm{p}<0.001$ ), peak torque generated by operated knee extensors $\left(76.17 \pm 30.08\right.$ vs. $83.36 \pm 25.46 \mathrm{~N} \cdot \mathrm{m} \cdot \mathrm{kg}^{-1} \mathrm{BW}$; $\mathrm{p}<0.001$ ), peak torque generated by surgical knee flexors $\left(47.66 \pm 15.13 \mathrm{~N} \cdot \mathrm{m} \cdot \mathrm{kg}^{-1} \mathrm{BW}\right.$ vs. $50.95 \pm 13.60 \mathrm{~N} \cdot \mathrm{m} \cdot \mathrm{kg}^{-1} \mathrm{BW}$; $\mathrm{p}=0.002)$, and the WOMAC-stiffness score $(2.92 \pm 1.36$ vs. $1.95 \pm 0.91 ; \mathrm{p}<0.001)$ improved significantly between the preoperative and 3-month postoperative measurements, respectively.

Postoperative outcomes of the unilateral and bilateral TKA groups with respect to objective performance-based physical function, self-reported physical function, pain, and quality of life

Differences in the postoperative physical function, pain, and quality of life outcomes between the groups are presented in Table 3. Time course of various functional measurements differed between the unilateral and bilateral TKA groups. At 1 month post-surgery, there were significant differences between the unilateral and bilateral TKA groups with respect to SCT ascent (15.52 \pm 4.79 vs. $18.34 \pm 5.76$ seconds, respectively; $\mathrm{p}=0.001)$, SCT descent ( $16.37 \pm 5.09$ vs. $17.50 \pm 6.06$ seconds, respectively; $\mathrm{p}<0.001)$, TUG test $(10.91 \pm 2.55$ vs. $12.12 \pm 3.58$ seconds, respectively; $\mathrm{p}=0.009)$, and VAS $(3.52 \pm 1.28$ vs. $4.04 \pm 0.97$, respectively; $\mathrm{p}=0.004$ ) scores. The unilateral TKA group showed significant improvement in the 6MWT (preoperative $428.82 \pm 97.54$ vs. postoperative $474.91 \pm 84.10$ $\mathrm{m} ; \mathrm{p}=0.001$ ) at 3 months post-surgery. This group also showed a significant difference in the WOMAC-function score at 1 month (preoperative $25.22 \pm 8.66$ vs. postoperative 28.39 $\pm 7.98 ; \mathrm{p}=0.024$ ) and 3 months postoperatively (preoperative $17.65 \pm 8.39$ vs. postoperative 20.82 \pm 7.39 ; $\mathrm{p}<0.001)$.

\section{DISCUSSION}

TKA is becoming more common; therefore, it is important to identify the most effective rehabilitation program to optimize patient care. According to several studies, 
Table 2. Postoperative outcomes of performance-based and self-reported physical function, pain and quality of life by repeated measure of ANOVA

\begin{tabular}{|c|c|c|c|}
\hline & \multirow{2}{*}{ Preoperation } & \multicolumn{2}{|c|}{ Postoperation } \\
\hline & & 1 month & 3 months \\
\hline SCT-ascent (s) & $14.14 \pm 5.71$ & $16.41 \pm 5.26^{\text {a) }}$ & $10.82 \pm 3.86^{\mathrm{a}, \mathrm{b})}$ \\
\hline SCT descent (s) & $16.90 \pm 5.81$ & $17.67 \pm 6.35$ & $12.13 \pm 4.03^{\mathrm{a}, \mathrm{b})}$ \\
\hline $6 \mathrm{MWT}(\mathrm{m})$ & $314.48 \pm 100.60$ & $366.65 \pm 81.40^{\mathrm{a})}$ & $444.04 \pm 95.36^{\mathrm{a}, \mathrm{b})}$ \\
\hline TUG (s) & $12.00 \pm 3.45$ & $11.28 \pm 2.94^{\mathrm{a})}$ & $9.31 \pm 1.75^{\mathrm{a}, \mathrm{b})}$ \\
\hline PT Ex of surgical knee $\left(\mathrm{N} \cdot \mathrm{m} \cdot \mathrm{kg}^{-1} \mathrm{BW}\right)$ & $76.17 \pm 30.08$ & $54.37 \pm 21.46^{\mathrm{a})}$ & $83.36 \pm 25.46^{\mathrm{a}, \mathrm{b})}$ \\
\hline PT Flx of surgical knee $\left(\mathrm{N} \cdot \mathrm{m} \cdot \mathrm{kg}^{-1} \mathrm{BW}\right)$ & $47.66 \pm 15.13$ & $46.57 \pm 14.02$ & $50.95 \pm 13.60^{\mathrm{a}, \mathrm{b})}$ \\
\hline Gait speed $(\mathrm{m} / \mathrm{s})$ & $0.89 \pm 0.17$ & $1.02 \pm 0.15^{\mathrm{a})}$ & $1.22 \pm 0.76^{\mathrm{a}, \mathrm{b})}$ \\
\hline ROM-knee flexion & $127.72 \pm 13.32$ & $114.36 \pm 15.16^{a)}$ & $121.49 \pm 12.65^{\mathrm{a}, \mathrm{b})}$ \\
\hline ROM-knee extension & $-7.1 \pm 5.88$ & $-2.45 \pm 4.40^{\mathrm{a})}$ & $-6.76 \pm 5.37^{\mathrm{b})}$ \\
\hline WOMAC-pain & $9.58 \pm 3.16$ & $6.57 \pm 2.5^{\mathrm{a})}$ & $4.7 \pm 2.15^{\mathrm{a}, \mathrm{b})}$ \\
\hline WOMAC-stiffness & $2.92 \pm 1.36$ & $2.68 \pm 1.09$ & $1.95 \pm 0.91^{\mathrm{a}, \mathrm{b})}$ \\
\hline WOMAC-function & $29.58 \pm 8.83$ & $26.29 \pm 8.54^{\text {a) }}$ & $18.72 \pm 8.16^{\mathrm{a}, \mathrm{b})}$ \\
\hline VAS & $6.98 \pm 1.70$ & $3.67 \pm 1.21^{\mathrm{a})}$ & $2.50 \pm 2.21^{\mathrm{a}, \mathrm{b})}$ \\
\hline EQ-5D & $0.58 \pm 0.15$ & $0.73 \pm 0.06^{\mathrm{a})}$ & $0.81 \pm 0.08^{\mathrm{a}, \mathrm{b}}$ \\
\hline
\end{tabular}

Values are presented as mean \pm standard deviation.

SCT, stair climbing test; 6MWT, 6-minute walk test; TUG, Timed Up and Go test; PT, peak torque; BW, body weight; Ex, extensor; Flx, flexor; ROM, range of motion; WOMAC, Western Ontario McMaster Universities Osteoarthritis Index; VAS, visual analog scale; EQ-5D, EuroQOL 5 dimensions.

${ }^{a)} \mathrm{p}<0.05$, significant difference between preoperatively and 1 month postoperatively, between preoperatively and 3 months postoperatively.

${ }^{b)} \mathrm{p}<0.05$, significant difference between 1 month postoperatively and 3 months postoperatively.

critical pathways reduce the length of hospital stay without increasing clinical complications; also, patients seem more inclined to make use of rehabilitation services $[27,28]$.

To the best of our knowledge, this study is the first to report on both physical and self-reported, short-term functional outcomes following the application of a critical pathway for early and inpatient TKA rehabilitation in Korea. Comparing other critical pathway programs, our critical pathway started on the 4 days of operation day, and patients were transferred to rehabilitation department at 2 weeks of operation day and trained for 2 weeks to achieve early intensive rehabilitation in terms of starting time and concentration. In addition, we have a strong advantage of using individualized and advanced equipment and evaluating the effects on various performance based physical function and self-reported physical function outcomes. The results showed significant improvement in a range of performance-based and self-reported physical functions, pain, and quality of life at 1 month and 3 months post-TKA.
Many studies have examined rehabilitation outcomes after TKA, and benefits and drawbacks have been reported. For example, Buhagiar et al. [10] conducted a randomized clinical trial of 165 unilateral TKA patients and compared an intensive inpatient rehabilitation group (which received physical therapy twice daily for 10 days) with a monitored home-based therapy group. Neither groups showed improved mobility at 26 weeks post-TKA. In contrast, our study recorded significant improvements in physical function (6MWT and EQ-5D scores) within a short period of time, although it is difficult to compare our study directly with that study, as that study was a randomized controlled trial. In addition, another study followed up 16 unilateral TKA patients who underwent early and highly intensive rehabilitation for a total of 25 visits over 12 weeks; patients showed positive results in terms of the SCT, TUG, and 6MWT scores at 3.5, 6.5, and 12 weeks post-TKA [7]. The rehabilitation program included both weight-bearing and non-weight-bearing exercises (such as quadriceps setting, straight leg raises, hip abduction, and hamstring curls with increasing resistance), 
Table 3. Postoperative outcomes of physical performance, physical function, pain and quality of life: subgroup analysis between unilateral and bilateral TKA groups

\begin{tabular}{|c|c|c|c|}
\hline & \multirow{2}{*}{ Preoperation } & \multicolumn{2}{|c|}{ Postoperation } \\
\hline & & 1 month & 3 months \\
\hline \multicolumn{4}{|c|}{ SCT-ascent (s) } \\
\hline Unilateral & $14.33 \pm 5.90$ & $15.52 \pm 4.79$ & $11.12 \pm 4.16^{\mathrm{a}, \mathrm{b})}$ \\
\hline Bilateral & $13.78 \pm 5.38$ & $18.34 \pm 5.76^{\mathrm{a}, \mathrm{c})}$ & $\left.10.27 \pm 3.17^{\mathrm{a}, \mathrm{b}}\right)$ \\
\hline \multicolumn{4}{|c|}{ SCT descent (s) } \\
\hline Unilateral & $16.66 \pm 5.71$ & $16.37 \pm 5.09$ & $\left.12.29 \pm 3.97^{\mathrm{a}, \mathrm{b}}\right)$ \\
\hline Bilateral & $17.50 \pm 6.06$ & $17.50 \pm 6.06^{a)}$ & $11.90 \pm 4.21^{\mathrm{a}, \mathrm{b})}$ \\
\hline \multicolumn{4}{|l|}{$6 \mathrm{MWT}(\mathrm{m})$} \\
\hline Unilateral & $311.63 \pm 99.77$ & $367.56 \pm 86.54^{\text {a) }}$ & $474.91 \pm 84.10^{\mathrm{a}, \mathrm{b})}$ \\
\hline Bilateral & $318.60 \pm 103.68$ & $363.25 \pm 70.90^{\mathrm{a})}$ & $428.82 \pm 97.54^{a, b, c)}$ \\
\hline \multicolumn{4}{|l|}{ TUG (s) } \\
\hline Unilateral & $12.00 \pm 3.38$ & $10.91 \pm 2.55^{\text {a) }}$ & $9.38 \pm 1.86^{\mathrm{a}, \mathrm{b})}$ \\
\hline Bilateral & $12.05 \pm 3.67$ & $12.12 \pm 3.58^{\mathrm{a}, \mathrm{c})}$ & $9.17 \pm 1.50^{\mathrm{a}, \mathrm{b})}$ \\
\hline \multicolumn{4}{|c|}{ PT Ex of surgical knee $\left(\mathrm{N} \cdot \mathrm{m} \cdot \mathrm{kg}^{-1} \mathrm{BW}\right)$} \\
\hline Unilateral & $72.50 \pm 27.80$ & $53.00 \pm 21.70^{\mathrm{a})}$ & $80.71 \pm 25.24^{\mathrm{a}, \mathrm{b})}$ \\
\hline Bilateral & $78.44 \pm 33.26$ & $57.69 \pm 22.42^{\mathrm{a})}$ & $89.55 \pm 27.03^{\mathrm{a}, \mathrm{b})}$ \\
\hline \multicolumn{4}{|c|}{ PT Flx of surgical knee $\left(\mathrm{N} \cdot \mathrm{m} \cdot \mathrm{kg}^{-1} \mathrm{BW}\right)$} \\
\hline Unilateral & $49.07 \pm 14.84$ & $48.52 \pm 13.30$ & $53.07 \pm 13.89^{\mathrm{a}, \mathrm{b})}$ \\
\hline Bilateral & $45.87 \pm 14.58$ & $46.48 \pm 15.08$ & $50.53 \pm 13.71^{\mathrm{a}, \mathrm{b})}$ \\
\hline \multicolumn{4}{|c|}{ Gait speed $(\mathrm{m} / \mathrm{s})$} \\
\hline Unilateral & $0.88 \pm 0.17$ & $1.02 \pm 0.15^{\mathrm{a})}$ & $1.23 \pm 0.91^{\mathrm{a}, \mathrm{b})}$ \\
\hline Bilateral & $0.90 \pm 0.18$ & $1.02 \pm 0.15^{\mathrm{a})}$ & $1.19 \pm 0.21^{\mathrm{a}, \mathrm{b})}$ \\
\hline \multicolumn{4}{|c|}{ ROM-knee flexion } \\
\hline Unilateral & $127.56 \pm 11.04$ & $114.10 \pm 17.71^{\mathrm{a})}$ & $122.35 \pm 11.69^{\mathrm{a}, \mathrm{b})}$ \\
\hline Bilateral & $129.68 \pm 9.92$ & $115.12 \pm 11.90^{\mathrm{a})}$ & $122.35 \pm 12.88^{\mathrm{a}, \mathrm{b})}$ \\
\hline \multicolumn{4}{|c|}{ ROM-knee extension } \\
\hline Unilateral & $-7.63 \pm 5.80$ & $-3.75 \pm 5.25^{a)}$ & $-7.91 \pm 5.52^{c)}$ \\
\hline Bilateral & $-7.58 \pm 6.23$ & $-1.23 \pm 3.02^{\mathrm{a})}$ & $-6.63 \pm 5.42^{\mathrm{c})}$ \\
\hline \multicolumn{4}{|c|}{ WOMAC-pain } \\
\hline Unilateral & $9.53 \pm 3.07$ & $6.33 \pm 2.53^{\mathrm{a})}$ & $4.40 \pm 2.19^{\mathrm{a}, \mathrm{b})}$ \\
\hline Bilateral & $9.75 \pm 3.36$ & $7.02 \pm 2.37^{\mathrm{a})}$ & $5.20 \pm 2.01^{a, b)}$ \\
\hline \multicolumn{4}{|c|}{ WOMAC-stiffness } \\
\hline Unilateral & $3.10 \pm 1.53$ & $2.69 \pm 1.10^{\mathrm{a})}$ & $1.82 \pm 0.92^{\mathrm{a}, \mathrm{b})}$ \\
\hline Bilateral & $2.65 \pm 0.95$ & $2.68 \pm 1.07$ & $2.19 \pm 0.81^{\mathrm{a}, \mathrm{b})}$ \\
\hline \multicolumn{4}{|c|}{ WOMAC-function } \\
\hline Unilateral & $29.35 \pm 8.72$ & $25.22 \pm 8.66^{\text {a) }}$ & $17.65 \pm 8.39^{\mathrm{a}, \mathrm{b})}$ \\
\hline Bilateral & $30.26 \pm 9.10$ & $28.39 \pm 7.98^{\mathrm{a}, \mathrm{c} c}$ & $20.82 \pm 7.39^{\mathrm{a}, \mathrm{b}, \mathrm{c})}$ \\
\hline \multicolumn{4}{|l|}{ VAS } \\
\hline Unilateral & $7.03 \pm 1.60$ & $3.52 \pm 1.28^{\mathrm{a})}$ & $2.55 \pm 1.34^{\mathrm{a}, \mathrm{b})}$ \\
\hline Bilateral & $6.93 \pm 1.85$ & $4.04 \pm 0.97^{\mathrm{a}, \mathrm{c})}$ & $2.41 \pm 0.93^{\mathrm{a}, \mathrm{b})}$ \\
\hline
\end{tabular}


Table 3. Continued

\begin{tabular}{lccc} 
& Preoperation & \multicolumn{2}{c}{ Postoperation } \\
\cline { 3 - 4 } & & 1 month & 3 months \\
\hline EQ-5D & & & \\
Unilateral & $0.59 \pm 0.15$ & $0.74 \pm 0.64^{\mathrm{a})}$ & $\left.0.81 \pm 0.08^{\mathrm{a}, \mathrm{b}}\right)$ \\
Bilateral & $0.56 \pm 0.16$ & $0.72 \pm 0.04^{\mathrm{a})}$ & $0.80 \pm 0.08^{\mathrm{a}, \mathrm{b})}$ \\
\hline
\end{tabular}

Values are presented as mean \pm standard deviation.

TKA, total knee arthroplasty; SCT, stair climbing test; 6MWT, 6-minute walk test; TUG, Timed Up and Go test; PT, peak torque; BW, body weight; Ex, extensor; Flx, flexor; ROM, range of motion; WOMAC, Western Ontario McMaster Universities Osteoarthritis Index; VAS, visual analog scale; EQ-5D, EuroQOL 5 dimensions.

${ }^{a)} \mathrm{p}<0.05$, significant difference between preoperatively and 1 month postoperatively, between preoperatively and 3 months postoperatively.

${ }^{b} \mathrm{p}<0.05$, significant difference between 1 month postoperatively and 3 months postoperatively.

${ }^{c)} \mathrm{p}<0.05$, significant difference between unilateral and bilateral TKA groups.

as did our study. However, we report superior outcomes for TUG and 6MWT scores at 3 months post-surgery; the SCT scores were similar. In addition, we initiated early rehabilitation at 2 weeks post-TKA and a period of 2 weeks, with significant improvements.

Gait speed is a useful tool for measuring a patient's physical function. A previous study reported 24 patients who underwent conventional rehabilitation after TKA; gait speed was slow until 3 months post-TKA, and at 6 months, it was similar to that recorded pre-TKA [29]. In contrast, our data show a linear improvement up to 3 months post-TKA. We believe that this finding is due to the intensive program based on the critical pathway in our study.

Various physical outcomes are used before and after discharge from hospital to assess functional recovery after TKA; however, they provide limited information. Thus, functional variables such as pain-VAS and WOMAC-function scores are effective tools for estimating pain and quality of life after TKA [30]. Consistent with our findings, Moffet et al. [31] demonstrated that subjects from an intensive functional rehabilitation group showed faster recovery and increased ability to perform daily activities with less pain, stiffness, and difficulty than conventional group following TKA. Furthermore, a study found that leg press power is more closely related to selfreported and performance-based outcomes than knee extensor strength [32]. In contrast, other studies report a more variable relationship between patient reported function and muscle strength $[33,34]$.

In terms of postoperative quadriceps muscle strength, we found that our protocol had a marked effect. Judd et al. [29] reported the results of 24 patients who underwent conventional post-TKA rehabilitation; compared with the preoperative value, there was a $42 \%$ decrease in the peak toque generated by operated knee extensors at 1 month post-TKA and $34 \%$ decrease in that generated by the knee flexors. Muscle strength did not return to preoperative levels until 3 months post-TKA. In contrast, we found only a $29 \%$ and $3 \%$ decrease in the peak toque generated by the surgical knee extensors and flexors, respectively; we also found that quadriceps muscle strength was significantly better at 3 months post-TKA than at pre-TKA. Within the first month post-TKA, the knee extensors and flexors were at their weakest (compared with pre-, post, and 1-year post-TKA) [35]. Such muscle weakness is predominantly due to the failure of spontaneous muscle activation, reduced ROM, and muscle atrophy [36]. This emphasizes the importance of rehabilitation strategies focusing on muscle strengthening during the early period after TKA; minimal reductions in early quadriceps muscle strength lead to improvements in several physical performance factors. Also, increased muscle strength has a positive effect on the SCT.

We found that the unilateral and bilateral TKA groups reported time differences with respect to functional measures, particularly in SCT, TUG, and VAS scores at 1 month, the 6MWT at 3 months, and WOMAC-function scores at 1 month and 3 months post-surgery. Several factors may be involved in these results, and presumably, the quadriceps strength of the non-surgical side and VAS score may affect performance. Iwata et al. reported that the VAS score and strength of quadriceps of the nonsurgical side were significantly associated with gait speed 
and TUG test [37]. A study by Valtonen et al. [38] on 56 patients with end stage knee OA reported that muscle power of the extensor and flexor of the knee on the nonsurgical side was independently associated with SCT. However, in contrast to our results, Huang et al. [39]conducted a comparative analysis of the unilateral and bilateral groups at 6 months post-TKA and showed similar functional improvement in pain, stiffness, function, and total WOMAC scores without significant difference between the two groups. However, that study only evaluated WOMAC scores at 6 months postoperatively, and it cannot be directly compared with our study as we evaluated various performance-based and self-reported physical functions at 1 - and 3 months post-TKA.

This study had some limitations. First, it was a retrospective cohort study with no control group; therefore, we could not compare our patients with those who did not receive early intensive rehabilitation. More research regarding the relationship between postoperative functional outcomes and the effects of our critical pathway program are required. Second, the study may suffer selection bias as only subjects followed up from pre-TKA to 3 months post-TKA were included. Therefore, longterm prospective comparative randomized control trial studies are needed. In conclusion, this study confirmed that patients who underwent early intensive rehabilitation within 2 weeks post-TKA via a critical care pathway showed significant improvements in various functional measures during the first 3 months post-TKA, although intergroup differences were observed in some functional outcome measures.

\section{CONFLICT OF INTEREST}

No potential conflict of interest relevant to this article was reported.

\section{ACKNOWLEDGMENTS}

This work was supported by the 2019 education, research and student guidance grant funded by Jeju National University.

\section{AUTHOR CONTRIBUTION}

Conceptualization: Kim BR, Kim JH. Methodology: Kim
BR, Kim JH, Han EY, Lee SY. Formal analysis: Kim BR, Kim JH. Project administration: Kim BR, Kim JH, Han EY, Kim SR, Nam KW, Lee SY, Kim WB. Visualization: Kim BR, Kim JH, Han EY, Kim SR, Nam KW, Lee SY, Kim WB. Writing - original draft: Kim BR, Kim JH. Writing - review and editing: Kim BR, Kim JH. Approval of final manuscript: all authors.

\section{REFERENCES}

1. Peat G, McCarney R, Croft P. Knee pain and osteoarthritis in older adults: a review of community burden and current use of primary health care. Ann Rheum Dis 2001;60:91-7.

2. Cushnaghan J, Bennett J, Reading I, Croft P, Byng P, Cox $\mathrm{K}$, et al. Long-term outcome following total knee arthroplasty: a controlled longitudinal study. Ann Rheum Dis 2009;68:642-7.

3. Ethgen O, Bruyere O, Richy F, Dardennes C, Reginster JY. Health-related quality of life in total hip and total knee arthroplasty. A qualitative and systematic review of the literature. J Bone Joint Surg Am 2004;86:963-74.

4. Xie F, Lo NN, Pullenayegum EM, Tarride JE, O'Reilly DJ, Goeree R, et al. Evaluation of health outcomes in osteoarthritis patients after total knee replacement: a two-year follow-up. Health Qual Life Outcomes 2010; 8:87.

5. Kehlet H, Thienpont E. Fast-track knee arthroplasty: status and future challenges. Knee 2013;20 Suppl 1: S29-33.

6. Jakobsen TL, Kehlet H, Husted H, Petersen J, Bandholm T. Early progressive strength training to enhance recovery after fast-track total knee arthroplasty: a randomized controlled trial. Arthritis Care Res (Hoboken) 2014;66:1856-66.

7. Bade MJ, Stevens-Lapsley JE. Early high-intensity rehabilitation following total knee arthroplasty improves outcomes. J Orthop Sports Phys Ther 2011;41:932-41.

8. Bade MJ, Kohrt WM, Stevens-Lapsley JE. Outcomes before and after total knee arthroplasty compared to healthy adults. J Orthop Sports Phys Ther 2010;40:55967.

9. Walsh M, Woodhouse LJ, Thomas SG, Finch E. Physical impairments and functional limitations: a comparison of individuals 1 year after total knee arthroplasty with control subjects. Phys Ther 1998;78:248- 
58.

10. Buhagiar MA, Naylor JM, Harris IA, Xuan W, Kohler F, Wright R, et al. Effect of inpatient rehabilitation vs a monitored home-based program on mobility in patients with total knee arthroplasty: the HIHO randomized clinical trial. JAMA 2017;317:1037-46.

11. Kang JH, Lee G, Kim KE, Lee YK, Lim JY. Determinants of functional outcomes using clinical pathways for rehabilitation after hip fracture surgery. Ann Geriatr Med Res 2018;22:26-32.

12. Duncan CM, Moeschler SM, Horlocker TT, Hanssen AD, Hebl JR. A self-paired comparison of perioperative outcomes before and after implementation of a clinical pathway in patients undergoing total knee arthroplasty. Reg Anesth Pain Med 2013;38:533-8.

13. Ribinik P, Le Moine F, de Korvin G, Coudeyre E, Genty M, Rannou F, et al. Physical and rehabilitation medicine (PRM) care pathways: "patients after total knee arthroplasty". Ann Phys Rehabil Med 2012;55:533-9.

14. van der Sluis G, Goldbohm RA, Elings JE, Nijhuis-van der Sanden MW, Akkermans RP, Bimmel R, et al. Preoperative functional mobility as an independent determinant of inpatient functional recovery after total knee arthroplasty during three periods that coincided with changes in clinical pathways. Bone Joint J 2017; 99-B:211-7.

15. Ayalon O, Liu S, Flics S, Cahill J, Juliano K, Cornell CN. A multimodal clinical pathway can reduce length of stay after total knee arthroplasty. HSS J 2011;7:9-15.

16. Lenssen AF, Crijns YH, Waltje EM, van Steyn MJ, Geesink RJ, van den Brandt PA, et al. Efficiency of immediate postoperative inpatient physical therapy following total knee arthroplasty: an RCT. BMC Musculoskelet Disord 2006;7:71.

17. Almeida GJ, Schroeder CA, Gil AB, Fitzgerald GK, Piva SR. Interrater reliability and validity of the stair ascend/descend test in subjects with total knee arthroplasty. Arch Phys Med Rehabil 2010;91:932-8.

18. Enright PL, McBurnie MA, Bittner V, Tracy RP, McNamara R, Arnold A, et al. The 6-min walk test: a quick measure of functional status in elderly adults. Chest 2003;123:387-98.

19. Podsiadlo D, Richardson S. The timed "Up \& Go": a test of basic functional mobility for frail elderly persons. J Am Geriatr Soc 1991;39:142-8.

20. Bugane F, Benedetti MG, Casadio G, Attala S, Biagi F,
Manca M, et al. Estimation of spatial-temporal gait parameters in level walking based on a single accelerometer: validation on normal subjects by standard gait analysis. Comput Methods Programs Biomed 2012;108:129-37.

21. Maffiuletti NA, Bizzini M, Desbrosses K, Babault N, Munzinger U. Reliability of knee extension and flexion measurements using the Con-Trex isokinetic dynamometer. Clin Physiol Funct Imaging 2007;27:346-53.

22. Thorstensson A, Karlsson J. Fatiguability and fibre composition of human skeletal muscle. Acta Physiol Scand 1976;98:318-22.

23. Cibere J, Bellamy N, Thorne A, Esdaile JM, McGorm $\mathrm{KJ}$, Chalmers A, et al. Reliability of the knee examination in osteoarthritis: effect of standardization. Arthritis Rheum 2004;50:458-68.

24. Bellamy N, Buchanan WW, Goldsmith $\mathrm{CH}$, Campbell J, Stitt LW. Validation study of WOMAC: a health status instrument for measuring clinically important patient relevant outcomes to antirheumatic drug therapy in patients with osteoarthritis of the hip or knee. J Rheumatol 1988;15:1833-40.

25. Carlsson AM. Assessment of chronic pain. I. Aspects of the reliability and validity of the visual analogue scale. Pain 1983;16:87-101.

26. Kim MH, Cho YS, Uhm WS, Kim S, Bae SC. Crosscultural adaptation and validation of the Korean version of the EQ-5D in patients with rheumatic diseases. Qual Life Res 2005;14:1401-6.

27. Benedetti MG, Sarti D, Stagni SB, Mariani E. Setting, clinical pathways, fast-track and rehabilitation following primary knee arthroplasty: a literature review. Open Rehabil J 2015;8:17-24.

28. Pennington JM, Jones DP, McIntyre S. Clinical pathways in total knee arthroplasty: a New Zealand experience. J Orthop Surg (Hong Kong) 2003;11:166-73.

29. Judd DL, Eckhoff DG, Stevens-Lapsley JE. Muscle strength loss in the lower limb after total knee arthroplasty. Am J Phys Med Rehabil 2012;91:220-6.

30. Salmon P, Hall GM, Peerbhoy D, Shenkin A, Parker C. Recovery from hip and knee arthroplasty: Patients' perspective on pain, function, quality of life, and wellbeing up to 6 months postoperatively. Arch Phys Med Rehabil 2001;82:360-6.

31. Moffet H, Collet JP, Shapiro SH, Paradis G, Marquis F, Roy L. Effectiveness of intensive rehabilitation on 
functional ability and quality of life after first total knee arthroplasty: a single-blind randomized controlled trial. Arch Phys Med Rehabil 2004;85:546-56.

32. Aalund PK, Larsen K, Hansen TB, Bandholm T. Normalized knee-extension strength or leg-press power after fast-track total knee arthroplasty: which measure is most closely associated with performance-based and self-reported function? Arch Phys Med Rehabil 2013;94:384-90.

33. Skoffer B, Dalgas U, Mechlenburg I, Soballe K, Maribo T. Functional performance is associated with both knee extensor and flexor muscle strength in patients scheduled for total knee arthroplasty: a cross-sectional study. J Rehabil Med 2015;47:454-9.

34. Brown K, Kachelman J, Topp R, Quesada PM, Nyland J, Malkani A, et al. Predictors of functional task performance among patients scheduled for total knee arthroplasty. J Strength Cond Res 2009;23:436-43.

35. Rossi MD, Brown LE, Whitehurst M. Knee extensor and flexor torque characteristics before and after uni- lateral total knee arthroplasty. Am J Phys Med Rehabil 2006;85:737-46.

36. Mizner RL, Petterson SC, Stevens JE, Vandenborne K, Snyder-Mackler L. Early quadriceps strength loss after total knee arthroplasty. The contributions of muscle atrophy and failure of voluntary muscle activation. J Bone Joint Surg Am 2005;87:1047-53.

37. Iwata A, Sano Y, Wanaka H, Yamamoto S, Yano Y, Iwata H. Different improvement trends in gait function and quadriceps strength early after total knee arthroplasty. J Phys Ther Sci 2019;31:57-62.

38. Valtonen AM, Poyhonen T, Manninen M, Heinonen A, Sipila S. Knee extensor and flexor muscle power explains stair ascension time in patients with unilateral late-stage knee osteoarthritis: a cross-sectional study. Arch Phys Med Rehabil 2015;96:253-9.

39. Huang YH, Lin C, Yang JH, Lin LC, Mou CY, Chiang KT, et al. No difference in the functional improvements between unilateral and bilateral total knee replacements. BMC Musculoskelet Disord 2018;19:87. 